\title{
Reetnización y legitimación de los indígenas kankuamo en los discursos de identidad*
}

\author{
CÉSAR AUGUSTO SÁNCHEZ CONTRERAS** \\ LUCÍA BUSTAMANTE VÉLEZ****
}

Recepción: 10 de marzo de 2020

Aprobación: 20 de junio de 2020

Forma de citar este artículo: Sánchez, C. A \& Bustamante, L. (2020). Reetnización y legitimación de los indígenas kankuamo en los discursos de identidad, Cuadernos de Lingüística Hispánica, (36), 19-38.

10.19053/0121053X.n36.2020.10875

* Artículo de investigación, producto del proyecto: "Identidad territorial: las prácticas culturales y la territorialidad en los discursos de identidad en los indígenas kankuamo del departamento del Cesar".

** Magíster en Territorio, Conflicto y Cultura, Universidad del Tolima; integrante del grupo Estudios Urbano-Regionales (GEURB); docente-investigador de la Universidad del Tolima. Correo electrónico: caugustosanchez@ut.edu.co Đhttps://orcid.org/00000002-8116-5936

*** Doctora en Lenguaje y Cultura, UPTC; integrante del grupo de investigación Corporación "Si Mañana Despierto" para la Creación e Investigación de la Literatura y las Artes; docente-investigadora de la Universidad Pedagógica y Tecnológica de Colombia. Correo electrónico: lucia.bustamante@uptc.edu.co Đhttps://orcid.org/0000-0002-7227-2019 


\title{
Resumen
}

El objetivo de este artículo es describir las características del proceso de reetnización de los indígenas kankuamo del departamento del Cesar, a través de los discursos de los actores que participaron en el proceso. Dichos discursos representan escenarios de legitimación, toda vez que se disponen como un instrumento de reconocimiento político y administrativo, que establece relaciones en espacios hegemónicos y de dominación. El análisis se propone desde la revisión de fuentes documentales, tales como los estudios etnológicos que apoyaron dicho reconocimiento institucional, documentos y registros audiovisuales del Resguardo Kankuamo que hacen parte de la memoria del proceso. Se discute el carácter político de la recuperación étnica y se establece que el discurso emerge como un dispositivo que se materializa en la reproducción social a través de los mecanismos de legitimación como el territorio, la identidad o la indigenidad.

Palabras clave: identidad, reetnización, análisis sociológico del discurso, indígenas kankuamo departamento del Cesar.

\section{Re-ethnization and Legitimation of Kankuamo Indigenous in Identity Discourses}

\begin{abstract}
This article aims to describe the characteristics of the process of re-ethnization of the Kankuamo Indigenous of Cesar state, through the speeches of the actors who participated in the process. These discourses represent scenarios of legitimacy since they are arranged as an instrument of political and administrative recognition, establishing relationships in hegemonic and domination spaces. The analysis is proposed from a documentary sources review, such as ethnological studies that supported such institutional recognition, documents and audiovisual records of the Kankuamo Reservation that are part of the process memory. The political nature of ethnic recovery is discussed, and it is established that the speech appears as a device that materializes in social reproduction through the mechanisms of legitimation such as territory, identity or indigenity.
\end{abstract}

Keywords: identity; re-ethnization; sociological analysis of discourse; Kankuamo Indigenous; Cesar state. 


\section{Ré-ethnicisation et légitimation du peuple indigène Kankuamo dans les discours identitaires}

\section{Résumé}

L'objectif de cet article est de décrire les caractéristiques du processus de réethnicisation du peuple indigène Kankuamo du département du Cesar, à travers les discours des acteurs qui y ont participé. Ces discours représentent des scénarios de légitimation, puisqu'ils constituent un instrument de reconnaissance politique et administrative, établissant des rapports dans des espaces hégémoniques et de domination. L'analyse est proposée à partir de la révision des sources documentaires, telles que les études ethnologiques qui ont soutenu cette reconnaissance institutionnelle, et les documents et enregistrements audiovisuels de la réserve de Kankuamo qui font partie de la mémoire du processus. La nature politique du rétablissement ethnique est débattue et le discours qui en apparait comme un dispositif qui se matérialise dans la reproduction sociale, à travers des mécanismes de légitimation tels que le territoire, l'identité ou l'indigénité, est établi.

Mots clés: identité ; ré-ethnisation ; analyse sociologique du discours ; peuple indigène de Kankuamo ; département du Cesar.

\section{Reetnização e legitimação de pessoas indígenas kankuamo em discursos de identidade}

\section{Resumo}

0 objetivo deste artigo é descrever as características do processo de re-etnização do povo indígena Kankuamo do departamento de Cesar, através dos discursos dos atores que participaram do processo. Esses discursos representam cenários de legitimação, pois são arranjados como instrumento de reconhecimento político e administrativo, estabelecendo relações nos espaços hegemônicos e de dominação. A análise é proposta a partir da revisão de fontes documentais, como os estudos etnológicos que apoiaram esse reconhecimento institucional, documentos e registros audiovisuais da Reserva Kankuamo que fazem parte da memória do processo. A natureza política da recuperação étnica é discutida e é estabelecido que o discurso surge como um dispositivo que se materializa na reprodução social por meio de mecanismos de legitimação como território, identidade ou indigenidade.

Palavras-chave: identidade; re-etnização; análise sociológica do discurso; povos indígenas de Kankuamo; departamento de Cesar. 


\section{Introducción}

La coyuntura política latinoamericana es un interesante escenario en el que surgen procesos de reivindicación por parte de movimientos sociales encabezados, en su gran mayoría, por indígenas y campesinos. En Ecuador, Chile, Bolivia y Colombia se presentan como acciones de movilización contra medidas político-económicas impuestas y planteadas por los gobiernos como respuesta a crisis globales de la economía neoliberal. En el caso colombiano, por ejemplo, estas acciones se expresaron en la considerable votación alcanzada por los movimientos políticos alternativos en las elecciones regionales del año 2019 (Calderón, 2019).

Dichos movimientos en América Latina han sido constantes desde la segunda mitad del siglo XX y para Colombia concretamente han posibilitado una notable respuesta con la participación política en el seno de la Asamblea Nacional Constituyente, que produjo la Constitución Política de 1991.

Teniendo en cuenta lo anterior, reconocemos que en el marco de los movimientos sociales surgen, como en el caso latinoamericano, acciones de recuperación de memorias ancestrales que, viéndose reivindicadas, tienen asidero en los escenarios políticoadministrativos del Estado. Ese escenario, de orden político principalmente, nos ofrece un necesario punto de partida en el contexto local sobre las posibilidades de inclusión en un proyecto de identidad nacional.

Los kankuamo son una de las cuatro comunidades indígenas de la Sierra Nevada, junto con los arhuacos, kogi y wiwa, y se constituyen en 12 comunidades según la configuración del resguardo (Resol. 12 del 2003, citada por Morales, 2011). Atánquez es la unidad territorial de mayor importancia y se reconoce como el principal referente territorial y administrativo de los kankuamo. La condición de comunidad indígena se da gracias al proceso de reetnización iniciado por líderes de la comunidad y académicos que optan por la recuperación de su identidad étnica y, a partir de 1994, proponen a los miembros de esta comunidad ante el Estado como indígenas; condición que es valorada hasta constituirse en resguardo, según se precisa en la Resolución 012 de 10 de abril de 2003 del entonces Instituto Colombiano de la Reforma Agraria INCORA (Morales, 2011).

Los elementos centrales de discusión sobre la reetnización son posibles en el contexto colombiano desde los movimientos sociales de reivindicación de derechos y participación indígena en la construcción de un nuevo escenario normativo. En el marco de la Asamblea Nacional Constituyente se fortalecen los escenarios políticos del 
movimiento indígena en Colombia y, a partir de 1993, se consolida un discurso nacional de diversidad y pluriculturalidad, que posiciona y legitima "nuevos actores" políticos y jurídicos en el Estado.

\section{Metodología}

La investigación describe acontecimientos y vincula relaciones históricas que se expresan en un conjunto de interacciones sociales y acumulación de procesos que se exponen como escenarios de interpretación. Lo anterior permite ubicarnos en el paradigma constructivista, el cual se sustenta en unas consideraciones epistemológicas de la cuales destacamos: la realidad se construye socialmente desde diversas formas de percibirla, el saber se erige por los participantes en el proceso, la investigación no es ajena a los valores del investigador y, por último, los resultados no pueden ser generados desconociendo el contexto y el tiempo. Por lo tanto, este paradigma permite describir la realidad a partir de los significados que se construyen en la interacción subjetiva. Así lo plantean Guba y Lincoln (2002):

Como la realidad se encuentra dentro de los significados que un grupo humano construye, la forma para acceder a ella es la interacción subjetiva entre los actores del fenómeno, donde el investigador no es un individuo ajeno, sino que es un miembro más con la misma importancia que el resto de los participantes. (Guba \& Lincoln, 2002; citados por Ramos, 2015, p. 14)

Ahora bien, el constructivismo permite aclarar vacíos que se expresan en preguntas ontológicas y cuya naturaleza no resuelve o edifica una única manera explicativa de la realidad.

Como principal estrategia de recolección y análisis propusimos los procedimientos y lecturas del análisis del discurso. Este tipo de investigación permitió abordar diferentes escenarios en los que se construye la identidad; por una parte, se pueden reconocer espacios discursivos que exponen el contexto desde una apuesta por la comprensión del orden social y, por otra, están los escenarios en los que el reconocimiento de la intersubjetividad social se da a través de discursos que contienen los sujetos que intervienen en la explicación social. Se planteó el análisis del discurso desde un punto de vista interpretativo, teniendo en cuenta que la interpretación sociológica del discurso consiste en establecer conexiones entre los discursos analizados y el espacio social en el que han surgido (Ruiz, 2009, p. 16)

Si bien podemos definir el discurso como cualquier práctica mediante la cual los sujetos dotan de sentido la realidad (Ruiz, 2009), también podemos reconocer la 
complejidad que ello implica: el discurso como práctica genera múltiples posibilidades como el sentido que le dan los sujetos a la acción.

De igual manera, desde Van Dijk (2009) nos apoyamos en la necesaria "forma" de exponer el discurso como un medio que representa y reproduce escenarios de poder y legitimación, y estos se vinculan a los intereses de los grupos o clases de dominación. El análisis del discurso surge como un tipo de investigación que se apoya en el enfoque propuesto y se precisa a través de elementos (orales y escritos) producidos por diferentes sujetos -líderes, representantes institucionales, representantes de la comunidad y actores sociales en general que hayan generado un tipo de discurso sobre los indígenas kankuamo-.

Metodológicamente, los discursos se organizaron teniendo en cuenta en qué espacio social se ubican, quién los produce y cuál es su relación con la comunidad. Se revisaron fuentes primarias como documentos oficiales, comunicaciones de la OIK, archivos de prensa representados en noticias, y la producción audiovisual, incluyendo canales informativos de la comunidad. Los discursos analizados tuvieron como ejes principales la historia cultural de la comunidad, el proceso de reconocimiento 0 legitimación y las condiciones y consecuencias del proceso de reetnización.

Los trabajos de Alicia Dussán y Gerardo Reichel-Dolmatoff (1977, 2012), Pumarejo y Morales (2003), Morales (2011), Castillo (2015) y Gros (2012) representan un discurso académico que está mediado por espacios de jerarquización y donde las dimensiones informativa, ideológica y como producto social se establecen a modo de marco de análisis de los textos como producto discursivo relevante.

\section{Resultados y discusión}

La reetnización es un concepto complejo que, a pesar de establecer su importancia en los elementos culturales o de corresponderse con características que constituyen lo que representa un grupo social, deja muchos interrogantes sobre su posibilidad. En el caso colombiano, la reetnización ha sido una herramienta que ha puesto en el debate académico las posturas antropológicas en las cuales son posibles los reconocimientos de comunidades que no perdieron totalmente sus características fundamentales a partir de los procesos de aculturación y que son necesarias formas de reivindicación o recuperación (Gros, 2012). Desde la perspectiva institucional, el Ministerio de Cultura manifiesta que

el término de reetnización ha sido acuñado por los Estudios Culturales y la Antropología para hacer referencia a los procesos generalizados en el mundo global, por parte de los grupos indígenas que han iniciado procesos de recuperación 
de tradiciones, de vestuarios, de su lengua, dietas, entre otros elementos de su identidad cultural tradicional. (Mincultura, 2019)

De tal manera que la reetnización, en cuanto elemento político, permite un avance en la legitimación de nuevos actores en la sociedad colombiana, se expone como un ejercicio de reivindicación cultural y apuesta desde los discursos académicos por espacios de participación y reconocimiento en el escenario administrativo nacional.

La reetnización, como instrumento de legitimación, permite el reconocimiento jurídico a las comunidades organizadas política y administrativamente. Este reconocimiento genera unos derechos particulares que se establecen como garantía de pertenencia al modelo sociopolítico que representa el Estado. Este punto es de gran importancia, porque permite evidenciar cómo funcionan las relaciones en escenarios hegemónicos y de dominación.

Para desarrollar lo anteriormente planteado, acudimos a los argumentos del investigador francés Christian Gros, quien expone no solo los conflictos políticos que implicó para el caso colombiano el reconocimiento de nuevos grupos étnicos, sino como todos esos tránsitos posibilitaron otros tipos de conflictos basados en la identidad y el territorio. Gros (2012) señala como fue el proceso de reconocimiento y "garantías" y lo que ello implicaba desde 1991; además, afirma que ser indígena en Colombia no solo tiene que ver con la identificación 0 adscripción histórica y cultural, sino que también se expresa en la legitimidad jurídica, la cual sustenta el reconocimiento de un derecho colectivo ejercido sobre un territorio. Dicho derecho generalmente es representado a través de la figura de cabildo u otra forma de autoridad propia, que extiende a sus integrantes toda una serie de garantías constitucionales, como atención gratuita en salud, derecho a una educación bilingüe y bicultural, acceso gratuito a la universidad, exención del servicio militar y de los impuestos sobre la tierra, derecho a ser juzgado según sus usos y costumbres dentro del seno de la comunidad y, desde la Constitución de 1991, derecho a transferencias del presupuesto nacional, derecho a una protección de su medioambiente y derecho a dos senadores elegidos a nivel nacional por circunscripción electoral especial (Gros, 2012, p. 65)

La territorialidad del poder local se expresa mediante el reconocimiento que hace el Estado de las formas de autoridad representadas en el ordenamiento jurídico de los nuevos grupos étnicos. Esta es la principal forma de reproducción social. Dicho análisis institucional, en términos sociológicos, se basa en el funcionamiento de las propiedades estructurales a partir del reconocimiento y mantenimiento de una figura que se expresa en la integración sistémica. Es decir, que en un análisis institucional de las estructuras sociales, como lo propone Giddens (2003), los elementos que dan posibilidad a las 
relaciones sociales se reproducen de forma arraigada y constante, mientras que en un análisis de las conductas de los sujetos la atención debe estar puesta en la manera en que se utilizan las relaciones sociales y a partir de qué motivaciones se establecen.

A partir del anterior argumento, señalamos que, a pesar de reconocer desde el funcionamiento institucional que las comunidades étnicas operan bajo unas relaciones definidas y unos principios establecidos a través de las figuras estructurales que reproducen legitimidad como el resguardo, estas figuras han generado algunos mecanismos de respuesta a escenarios de hegemonía institucional. La autonomía, cosmovisión y territorialidad han servido como elementos reguladores en el mantenimiento de una identidad.

De acuerdo con Castillo (2015), los movimientos indígenas de los sesenta y setenta del siglo XX en Colombia y en parte de América Latina se centraron en la reinvención de la identidad en una relación directa con el territorio, que se refleja en que:

el indígena había aprendido, desde el periodo colonial, que estaba "resguardado" en el resguardo. El derecho a la propiedad colectiva sobre la tierra le había permitido subsistir en una sociedad que lo despreciaba. El secreto de esto consistía en que en el resguardo ejercía una territorialidad tradicional; un control sobre un conjunto de recursos (tierra, agua, bosque) que le había garantizado, a pesar de todo, la continuidad de algunas instituciones muy importantes de su cultura. (Castillo, 2015, p. 93)

Sobre los kankuamo, Castillo (2015) expresa que, en este caso, la identidad se modifica 0 transforma de "negativa" a "positiva", puesto que se reconstruye a partir de una búsqueda por el reconocimiento público o externo. Resalta que este pueblo fue descrito en una famosa etnografía titulada Pueblo de Aritama, del antropólogo ReichelDolmatoff, como asimilado totalmente al campesinado (Castillo, 2015, p. 94).

Christian Gros (2012) dedica el segundo capítulo de su texto Políticas de la etnicidad. Identidad, Estado y modernidad a mostrar desde el caso colombiano lo que denomina identidades nuevas. Participó en los congresos indígenas que dieron origen al proceso de reconocimiento de los kankuamo y desde esa experiencia elaboró un escenario discursivo alrededor de la modernidad como eje del análisis en la construcción política de la identidad. Desde el capítulo señalado, Gros desarrolla su propuesta alrededor de una serie de preguntas o problemas centrales que se plantean de la siguiente manera; ¿Cómo y porqué se construye y reconstruye una identidad indígena y cuál puede ser el contenido de esta identidad bien presente en diferentes escenarios -sociales, culturales, y políticos- de América Latina? Y ise puede disolver el indígena en la modernidad? 
La propuesta de Gros (2012) liga la historia "moderna" de Colombia con los movimientos étnicos que son interferidos o surgen de dicho proceso. Expone características del movimiento indígena y reaviva la idea de instrumentalización o manipulación de la condición étnica 0 , de forma más general, de la etnicidad.

Reconocer el complejo efecto político de la identidad, pone de manifiesto la necesidad de hacer un análisis a las formas hegemónicas y de control que se establecen en los grupos sociales y las consecuencias que genera el constreñimiento estructural. Las razones que surgen de los actores sociales, sitúan las posibilidades de su acción en un rango menor por la interferencia de las estructuras sociales en las posibilidades que le plantea al actor 0 grupo social. En Giddens (2003), el anterior argumento se expresa con la propuesta de análisis del poder, el cual concibe como un medio para obtener que se hagan las cosas y, a pesar de no ser decisivo en la reproducción social, se manifiesta a través de "pugnas" o "luchas" por conseguir el control (Giddens, 2003, p. 309).

En el escenario de legitimación de los indígenas, el reconocimiento de los kankuamo surge como el principio de defensa para garantizar el funcionamiento de su organización social. Este es, por supuesto, un escenario político, pero es el único posible para la materialización de sus posibilidades como comunidad a partir de sus condiciones de vida.

Es en la importancia del territorio o, más bien, del resguardo, que se legitima la defensa de las condiciones de vida del indígena. Pero esta figura había sido un obstáculo para las intenciones del proyecto de nación mestiza propuesto por las élites liberales de la naciente república de Colombia desde finales del siglo XIX. Dicho proyecto se sustentaba en el pensamiento y doctrinas liberales que sostenían que la igualdad era una conquista de la humanidad en contra del Antiguo Régimen, y en esa premisa se fundaba el ejercicio de la libertad a través de la propiedad privada. Sin duda, dicha concepción en la conformación de un Estado moderno es contraria a la existencia de tierras no enajenables, comunales, y producto del orden colonial, de tal manera y de acuerdo con lo que señala Castillo (2005) en su tesis sobre etnicidad y nación, "este proyecto era incompatible con la existencia de resguardos y cacicazgos porque ello implicaba el ejercicio de la territorialidad, es decir, el control sobre gente, recursos y territorio" (p. 97).

El anterior contexto dio las bases para el surgimiento de un fuerte movimiento indígena y campesino, y formas de resistencia para la recuperación de los territorios. Se crearon dos entes de gran importancia para el movimiento social en Colombia, la Asociación de Nacional de Usuarios Campesinos (ANUC) y el Consejo Regional Indígena del Cauca (CRIC), y posteriormente la Organización Nacional Indígena de Colombia 
(ONIC), desde los cuales se desarrolló un intenso trabajo ideológico y práctico que se reflejó en un activismo fundamental para la creación de formas asociativas y cooperativas que tuvieron inicialmente impacto regional, pero que lograron posteriormente su consolidación como movimiento nacional. En dicho espacio se contemplaron, a su vez, unas formas de acción colectiva que se reflejaba en la lucha por la tierra, el reconocimiento de las figuras representativas de carácter político, como el cabildo, y la construcción y el reconocimiento de un pensamiento propio que diera posibilidades al derecho mayor: autoridad y territorio propios.

Es en el marco del fortalecimiento de los movimientos sociales y en el escenario del IV Congreso Nacional de Indígenas de 1993, cuando emerge la figura de los kankuamo, situados en el corregimiento de Atánquez del municipio de Valledupar.

\section{Bases para un análisis sociológico del discurso: ¿atanqueros o kankuamos?}

La principal referencia para cualquier estudio sobre los kankuamo es el denso trabajo etnográfico La gente de Aritama, realizado por Alicia Dussán y Gerardo ReichelDolmatoff (2012), como ya se anotó. Dicho trabajo ha sido la base para diferentes interpretaciones sobre esta comunidad, que fue presentada como campesina en la publicación exhibida inicialmente en inglés en el año de 1961, reimpresa en 1970 por la Universidad de Chicago y finalmente traducida al español y presentada en el año 2012 por la Pontificia Universidad Javeriana y ópera Máxima. De ese trabajo y otros realizados en la denominada región Andina surge una compilación presentada en 1977 por el Instituto Nacional de Cultura, la cual se compone de tres grandes capítulos, siendo el tercero el más esclarecedor sobre la manera en que los esposos Reichel-Dolmatoff reconocían la comunidad de Atánquez.

La gente de Aritama (2012) es un impresionante texto, que se estructura a partir de un contenido etnológico, caracterizado por una densa etnografía que contempla la interpretación a través de la descripción geográfica, la configuración demográfica, el reconocimiento de elementos biofisiológicos, las bases sociopsicológicas, la descripción de las relaciones sociales, la identificación de las formas de producción y la configuración cultural de los campesinos de Aritama (nombre ficticio que los antropólogos Dussán y Reichel--Dolmatoff le asignaron a Atánquez).

Si bien el texto se estructura detalladamente a partir de la relación que establecieron los autores con los miembros de la comunidad, estos se proponen resguardar el nombre real de Atánquez en la producción académica y es solo hasta 1977 cuando en Estudios Antropológicos (1977), exponen oficialmente en su tercera parte un capítulo 
denominado "La repartición de alimentos en una sociedad de transición”. Dicho capitulo es significativo, en la medida en que el discurso sobre los kankuamo aparece de manera directa sin seudónimos, pero fundamentalmente por reconocer las características que según el campo de conocimiento antropológico y experiencias de los autores hacían parte de la comunidad.

Ahora bien, la transición aparece en la obra de los antropólogos Dussán y Reichel-Dolmatoff muy cercana a la noción de desarrollo cultural. En este escenario, la cohesión y la solidaridad son sinónimos de transformación o modificación en el sentido de mejora en una comunidad en la que predominan procesos de aculturación y desintegración. En este caso, el intercambio aparece como necesario en la confirmación de los lazos de parentesco a través de algunos mecanismos de control social como la magia (1977, pp. 486-504). En las condiciones de vida características de la población, describen una comunidad basada en la agricultura rudimentaria, dependiente de la regularidad del clima y fundamentalmente de subsistencia. Describen a los habitantes de esta población como "muy poco trabajadores" comparados con la población campesina del norte del país, con una clara ausencia de acumulación de su producción agrícola, pero sí de distribución debido a sus características culturales (Dussán \& Reichel-Dolmatoff, 1977, p. 496).

Efectivamente, la población de Atánquez en la narrativa anterior está muy cercana a una descripción objetiva de su cultura y condiciones de vida, pero con aspectos valorativos implícitos al generar formas comparativas, sin un sentido peyorativo, sino más bien propositivo, para que el lector pudiese reconocer o comprender que se trataba de exponer las condiciones materiales de una sociedad que para los autores era considerada de transición. Es necesario reconocer además que las relaciones sociales y la forma de vida expuesta sobre la población de Atánquez se corresponden con la noción de sociedad campesina, a pesar de no estar definidas ni claramente establecidas las diferencias 0 características esenciales entre los diferentes grupos que configuran parte de la población rural de la región. Por supuesto que ser indígena 0 afrodescendiente supondría una marcada diferencia desde las costumbres, lenguas, indumentaria, expresiones 0 manifestaciones culturales; sin embargo, esta parte de la hoy denominada región Caribe se ha caracterizado por un complejo proceso étnico y cultural que sienta sus bases en el control territorial.

Según Van Dijk (2009), la mayoría de los libros de texto reproducen una cosmovisión nacionalista, etnocéntrica o racista, tanto de los demás pueblos como de los grupos de minorías étnicas. De igual manera, señala que los países del tercer mundo y las 
minorías (negras) suelen aparecer representados como "atrasados" en comparación con "nuestra" posición y "nuestro desarrollo", cuando no se los retrata como "primitivos", "holgazanes" y "estúpidos" (p. 105)

Ahora bien, la necesaria emergencia de la producción académica sobre la comunidad de Atánquez, permitió tener un punto de referencia sobre aspectos globales para comprender las dinámicas de una población que reconoce su historia y decide a partir de ella resignificarse y proponer la recuperación de una memoria indígena dentro de unos marcos sociales que se sustentan en el pagamento, los lugares sagrados, el Corpus Christi y el fortalecimiento de la territorialidad como elemento central de legitimación.

\section{El reconocimiento como base de legitimación}

Dos textos surgen en el campo académico a partir del proceso de reconocimiento a los indígenas kankuamo que se inicia oficialmente desde 1993: uno, escrito por Pumarejo y Morales (2003), quienes elaboran su tesis de pregrado en Antropología y representan parte del acompañamiento al proceso político, administrativo y cultural de reconocimiento oficial de los indígenas kankuamo y generan desde allí un documento base para dicha acción institucional; y un segundo texto, presentado por Morales (2011) como resultado de su tesis doctoral en Antropología Social, que se concentra en la descripción de los elementos que considera centrales en la condición étnica de los kankuamo, como lo son las festividades del Corpus Christi y los pagamentos.

El texto La recuperación de la memoria bistórica de los Kankuamo: un llamado de los antiguos siglos XX-XVIII, (2003), se plantea en tres grandes partes: Atánquez: el final comienzo Atánquez: la frontera entre el Valle y la Sierra; y Atánquez: la Sierra. Cada una de ellas construida desde la historia oral, procurando establecer una línea de tiempo basada en los hitos que dieron cuenta de las características del origen, la pérdida y la reaparición de la condición étnica del pueblo kankuamo. En el texto se teje un discurso elocuente pero también con matices políticos en la relación con otras culturas y territorios, fundamentalmente con Valledupar; además, propone la categoría central de identidad a través de toda una apuesta discursiva alrededor de la memoria. Dicho elemento se asocia a la noción de recuperación y es fundamental para el reto que genera el movimiento de reivindicación que se adscribe inicialmente en un grupo de habitantes de la zona, músicos, profesionales y organizadores del Festival Folclórico y Artesanal de la Sierra Nevada que se originó a mediados de los 80 .

Por su parte, el texto Los idiomas de la reetnización. Corpus Christiy pagamentos entre los indígenas Kankuamo de la Sierra Nevada de Santa Marta, de Patrick 
Morales (2011), es un detallado documento que expone desde una etnografía densa la importancia del Corpus Christi en la herencia histórica que representan los rituales y danzas que configuran esta festividad sincrética en Atánquez, además de los pagamentos, que se reconocen como lugares representativos o hitos fundacionales que se integran a la memoria colectiva del pasado indígena kankuamo. El texto busca consolidar los elementos que hicieron posible el reconocimiento institucional de los indígenas kankuamo en los ámbitos local y nacional, muestra parte del proceso organizativo que emerge como un movimiento que en reiteradas ocasiones es descrito por sus participantes como una lucha, y se concentra en una muy detallada representación del Corpus Christi como escenario festivo alrededor de sus participantes como los reproductores de una memoria asociada a la condición étnica y su cosmovisión.

Un elemento importante en el que Morales (2011) insiste, gira alrededor del problema territorial, que se constituye en el eje de todo reconocimiento étnico en Colombia, limita con las disputas de legitimación o autorreconocimiento por parte de actores o pequeños grupos que construyeron una identidad con la noción de sujetos campesinos. Al respecto señala:

El problema territorial, no obstante, no se inscribía solamente en el plano legal, pues la lectura de la dimensión territorial se proponía desde múltiples niveles. En efecto, según las delimitaciones ancestrales reivindicadas por algunos habitantes de la región, una gran parte de las tierras hoy ocupadas por los kogi, los ika y los wiwa de la vertiente suroriental pertenecen a los kankuamo. Una de las primeras tareas emprendidas por la OIK fue recoger a partir de múltiples versiones orales los límites del territorio ancestral kankuamo. Hay que anotar que todas se recortan y entrecruzan con las de los resguardos actualmente constituidos en la zona. Si las organizaciones indígenas de la Sierra reconocían el hecho de que muchas de las tierras hoy ocupadas habían sido de los atanqueros, la validación no la hacían desde el reconocimiento de los límites ancestrales reivindicados por los kankuamo, sino desde su propia experiencia histórica de colonización de tierras no explotadas y de negociación lenta y paciente con los habitantes de la región. (2011, p. 99)

Desde el texto también se puede inferir que uno de los principios de acuerdo sobre la legitimación en el proceso de reconocimiento se funda en la aceptación y valoración por parte de los otros grupos étnicos de la Sierra Nevada. Además de la cercanía con los forjadores del proceso de reconocimiento, la existencia del consenso que se reflejaba en los discursos de algunas autoridades indígenas kogui, ika o wiwa sobre la existencia de los kankuamo daban la posibilidad de una solución a los eventuales conflictos por el 
territorio. Pero, además, la importancia para el proceso de reconocimiento institucional que significó la participación directa de los antropólogos en el concepto de valoración de la etnicidad, se basó según los autores, en ir más allá de una comprobación de rasgos o una veracidad o falsedad del carácter étnico en el que insistía la institucionalidad. Morales (2011) lo resume de la siguiente manera:

El 'concepto' sobre el pueblo kankuamo elaborado para la DAI, basado en la investigación realizada por los antropólogos durante la tesis de pregrado, proponía una mirada sobre la identidad de los habitantes de la región más allá de aquella pregunta errónea sobre la cual se basaban estos dictámenes respecto a la veracidad o falsedad del carácter étnico de los pueblos en cuestión y sugería además considerar el pagamento como un hilo conductor de un proceso continuo de resignificación identitaria que parecía inscribirse desde diversos planos de la práctica social. La reconstrucción de la memoria de los habitantes de la región no se limitaba al plano estrictamente narrativo, pues a partir de las experiencias de los recorridos territoriales había sido posible comprobar la estrecha relación que existía entre territorio y memoria en un marco continuamente resignificado por la recuperación activa de los pagamentos. Asimismo, algunas de las construcciones narrativas sobre el pasado, estrechamente relacionados con el pagamento, reaparecían en el plano ritual y se articulaban de esta manera a relatos flexibles y plenamente vigentes entre los habitantes de la región. El pagamento no solo proponía un ámbito significativo desde el cual negociar las versiones y posiciones identitarias, sino que a la vez era el marco a partir del cual se inscribía desde el presente la cambiante relación con el pasado entre los habitantes de la región (p. 142).

Así, mientras emergían bases sólidas para el reconocimiento institucional, la iniciativa se fortalecía con los procesos organizativos que se sustentan en la creación de un espacio de convergencia denominado "Primer Congreso del Pueblo Indígena Kankuamo", celebrado en diciembre de 1993 en las poblaciones de Atánquez y Chemesquemena. Dicho Congreso se lleva a cabo después de la participación de algunos miembros de la comunidad en el IV Congreso Indígena Nacional, celebrado del 30 de agosto al 3 de septiembre del mismo año en Natagaima, Tolima (Gros, 2012, p. 59), donde presentaron a las organizaciones indígenas de Colombia su decisión de iniciar el proceso de recuperación de su indigenidad, decisión que fue apoyada y acompañada por la ONIC.

Como consecuencia del Primer Congreso kankuamo, la fortaleza del proceso se logra comenzando con la conformación de las autoridades de la comunidad y la creación de la Organización Indígena Kankuama (OIK); es así como para el 10 de junio de 1994 
se posesiona ante el alcalde de Valledupar el cabildo gobernador Jaime Arias, elegido en el marco del Congreso Kankuamo con el aval de las autoridades políticas y religiosas representadas en los mamos (Morales, p. 113). Así las cosas, los indígenas kankuamo, antes del reconocimiento del resguardo como acción de legitimación institucional en abril de 2003, tenían dos acciones jurídicas que daban vida ante el Estado colombiano a la comunidad; la primera, la posesión de los cabildos ante el representante del ente territorial, y la segunda, la vinculación en la Ley 99 del 22 de diciembre de 1993, donde, a efectos del manejo de la Corporación para el Desarrollo Sostenible de la Sierra Nevada de Santa Marta se le asignaba un cupo a los kankuamo.

Un factor importante, que hace parte de los elementos que configuran la identidad en términos políticos, son las condiciones representadas en la integración y los elementos comunes como el racial o el folclórico, es así como el festival folclórico se vincula con la anterior aseveración. Morales expone lo siguiente sobre el festival:

En ese orden de ideas, el festival buscaba así mismo posicionar y preservar una identidad de la región, acorde con las transformaciones producidas en el plano de las relaciones entre los actores locales. Este posicionamiento, sin embargo, se construyó claramente desde el terreno de lo folclórico; desde este registro se propuso, pues, inscribir a Atánquez como un lugar de mediación entre las culturas indígenas de la Sierra y la experiencia histórica de unos campesinos que contaban con un pasado indígena expresado en sus tradiciones folclóricas. No podía ser de otra manera, pues para los atanqueros que viven ahora en Valledupar, actores importantes en la organización y promoción del festival, resultaba difícil reivindicar una identidad indígena contemporánea luego de décadas de presentarse a sí mismos como mestizos integrados a las dinámicas sociales de las tierras bajas. Mientras tanto, si muchos en el pueblo eran prudentes a la hora de evocar la recuperación más allá de los límites del festival, la dinámica generada por el evento y las transformaciones legislativas consagradas en la reciente Constitución de 1991 no dejaron indiferentes a muchos líderes locales dispuestos a tomar en serio el asunto de una identidad indígena por redescubrir. (2011, p. 97)

Llama la atención el adjetivo de "identidad indígena contemporánea", puesto que, a pesar de no asignarlo a la comunidad, Morales (2011) lo plantea como un escenario de posibilidades frente al reconocimiento de sus integrantes. La anterior es posiblemente una observación simplista que hacemos desde el marco discursivo del autor en mención, pero intenta vincular los marcos que definen el campo discursivo académico y las constantes elucubraciones de surgen de allí. En últimas, estos elementos representan 
discursos hegemónicos con base en un tipo de conocimiento basado en la epistemología occidental.

Ahora bien, en otro tipo de discursos, ya desde la voz directa de los miembros de la comunidad, vemos como coinciden con los institucionales vinculados a la narración sobre el proceso de reconocimiento, pero también desde la experiencia de quienes participaron. Del documental "Somos Kankuamos" presentado en YouTube (2016) por el resguardo, rescatamos algunos fragmentos de entrevistas producidas a integrantes del cabildo indígena, donde expresan lo siguiente sobre el proceso de reetnización:

"Hay que recordar que nuestros mayores, nuestras madres, nuestros padres, nos dejaron un legado, el cual fue diezmado, desquebrajado por los españoles, por la iglesia que llegó en su momento".

"[...] el renacer Kankuamo junta una mayoría de mayores, jóvenes, profesores, religiosos, dentro de esos estaban los pastores, y ellos nos ayudaron a hacer eso".

"[...] a partir de los años ochenta, ochenta y seis, se realiza un primer encuentro que se llama encuentro cultural de la Sierra Nevada de Santa Marta, donde el pueblo Kankuamo a través de muchos hombre y mujeres comienzan a organizar algo que se llamó el festival folclórico, y allí se comenzó con el rescate de la música, la mochila y las danzas propias"

"En el año noventa y tres, apoyados y asesorados por unos hermanos representantes de la ONIC, vinieron y nos invitaron a un congreso nacional, en ese entonces era el cuarto congreso realizado en Natagaima Tolima, y allí estuvimos mirando la experiencia. Después de eso vinimos con ganas de hacer un congreso propio y lo hicimos en diciembre, de lo cual nació la Organización Indígena Kankuama". (Cabildo Indígena, 2016, 3:40-6:55)

Por su parte, la ONG Derecho, Justicia y Sociedad (Dejusticia) produce un interesante documental titulado "Resistencia Kankuama: desde el corazón del mundo" (2018); allí, el cabildo gobernador Jaime Arias expone la importancia y origen del proceso de recuperación:

[...] a partir del año 1993 se constituye la Organización Indígena Kankuama nuevamente, con el objetivo fundamental de recuperar y apropiar la identidad cultural, el fortalecimiento del gobierno propio y la autonomía, el afianzamiento y conciliación territorial y la consolidación de un modelo de desarrollo propio, en armonía con la madre naturaleza. Estamos también en un proceso de lucha y resistencia que a nosotros también como pueblo nos ha tocado enfrentar a lo largo de la historia, no solo en la época de la conquista y la colonización, sino 
luego cuando llegaron las bonanzas marimberas que nos afectaron a nosotros, vinieron las guerras de la violencia política, cuando se habló de la chulavita por ejemplo y luego el conflicto armado, ya al final que se conoce, el enfrentamiento de la guerrilla, acá hizo presencia las FARC el ELN, el EPL, con el ejercito o sea con la fuerza pública, y en medio de eso surgen los paramilitares también, y eso da como resultado más de 350 miembros del pueblo Kankuamo asesinados, en masacres en asesinatos selectivos, muchos miembros del pueblo Kankuamo desaparecidos y más de 500 familias desplazadas. Por eso nosotros sentimos la responsabilidad de cuidar y conservar o aportar desde nuestras ceremonias, no solo para nosotros sino para toda la humanidad. Nuestros padres espirituales Serankua y Seneka, nos dejaron en este territorio, pero también nos dejaron una misión muy importante, que fue cuidar a todos los seres de la naturaleza, o sea que nosotros somos responsables de cuidar todo eso y eso es una gran responsabilidad. Entonces nosotros no tener esa relación cultural con el territorio es no tener vida. (Dejusticia, 2018, 1:16-3:17)

Si bien la narración gira alrededor de las bases políticas del funcionamiento del resguardo y de la construcción del sujeto indígena, el gobernador Arias construye un relato vinculando los inconvenientes que le ha generado dicho reconocimiento a la comunidad al ser expuesta y vinculada al conflicto armado en Colombia, el cual se caracterizó históricamente por la "lucha por la tierra". Este es un importante aspecto para considerar, puesto que, si bien la legitimación institucional plantea el reconocimiento de la comunidad como un principio de derecho, al mismo tiempo genera acciones de deslegitimación a través de sectores de poder que propenden al sometimiento y control.

Resumiendo, los diferentes discursos que giran alrededor del proceso de reetnización de los indígenas kankuamo de la Sierra Nevada, se inscriben en diferentes escalas en la noción territorial y dan posibilidades a una desventaja en el sentido de interferencia en la configuración estructural de la comunidad. De tal manera que, en términos de lo que podemos proponer como emergencia del discurso hegemónico en la reproducción social a partir de: la existencia de la comunidad fundada en un discurso etnológico que expone a un grupo social distante de su origen étnico e histórico, pero cuya presencia le genera posibilidades; un discurso antropológico, cuya base etnográfica se sustenta en la memoria como base de recuperación; y un discurso inscrito en la descripción de manifestaciones culturales, cuya principal característica se asocia a la legitimación de saberes y comportamientos rituales como mecanismos de adscripción étnica. Teniendo en cuenta lo anterior, surge entonces la necesidad de proponer la vinculación de los escenarios discursivos hegemónicos con las formas de constreñimiento institucional que 
se hacen efectivas a través de una dualidad en las condiciones inadvertidas de la acción. Es decir, que, una vez establecidos los procesos de reconocimiento o legitimación de la comunidad desde la perspectiva o base institucional, en dicho proceso opera una serie de vínculos desde las estructuras sociales para incorporar y reproducir sus principios estructurales, los cuales están basados en la reproducción de recursos y reglas como base del orden social.

Ahora bien, el hecho de que en los discursos hegemónicos se reproduzcan formas institucionales de la acción, ello no implica que los grupos (actores sociales) no encuentren u originen mecanismos de reproducción de una conducta estratégica, entendida esta como la capacidad de generar acción hacia las estructuras. En el caso de los kankuamo, esta se ve reflejada en el espacio social que constituye el campo de poder; es decir, en los recursos que le dan posibilidades de pertenecer a un grupo específico con garantías institucionales y legítimas en el ordenamiento institucional.

\section{Conclusiones}

El artículo propone un análisis sobre los kankuamo a través de los discursos que los hicieron posible en el contexto nacional. Se describen sus características como comunidad, los discursos alrededor de su proceso de reindigenización o reetnización y sobre la forma en que estos posibilitaron la configuración de su identidad.

Se aborda el fenómeno de la reetnización desde unas interpretaciones que se han convertido en lugares comunes como: "la etnicidad es una reivindicación estratégica", o "la interpretación del pasado es moldeada por los intereses políticos" como señala Caviedes (2012).

Se establece que el discurso emerge como un dispositivo que se materializa en la reproducción social a través de los mecanismos de legitimación como el territorio, la identidad o la indigenidad. Estos elementos no necesariamente se establecen de forma directa en las relaciones sociales, sino que se vinculan a través de las conductas estratégicas e institucionales. En ese sentido es importante el análisis del discurso, puesto que puede alcanzar el objetivo que se plantea, esto es, mejorar nuestra comprensión y aumentar nuestro conocimiento de los fenómenos sociales.

La base del reconocimiento de los indígenas kankuamo se ha planteado desde el conocimiento antropológico, el cual, como herramienta institucional, se ha consolidado en las reivindicaciones étnicas y los procesos de adjudicación constitucional, lo que ha permitido reconocer estas acciones como producto de la estructura social. Bajo esa 
lógica, se debe mantener la vigilancia epistemológica, puesto que la legitimación sobre lo indígena puede ser pensada y pronunciada con base en un discurso académico que se nos presenta como el único posible y el único correcto.

\section{Referencias}

Cabildo Indígena del Resguardo Kuankamo. (2016, 11 de nov.). Somos Kankuamos. [Video]. YouTube. Recuperado de https://www.youtube.com/watch?v=6Tsjsze4fi8\&t=46s

Calderón, J. (2019, 16 abr.). Dinámicas, actores. Recuperado de https://www.celag.org/colombiadinamicas-actores-proximas-elecciones-regionales/\#

Castillo, L. C. (2005). El estado-nación pluriétnico y multicultural colombiano: la lucha por el territorio en la reimaginación de la nación y la reinvención de la indentidad étnica de negros e indígenas. (Tesis doctoral). Universidad Complutense de Madrid.

Castillo, L. C. (2015). Etnicidad y nación. El desafio de la diversidad en Colombia. Cali: Universidad del Valle.

Caviedes, M. (2012). Recuperado de https://revistas.unal.edu.co/index.php/maguare/article/ view/35326/39563.

Derecho, Justicia y Sociedad -Dejusticia-. (2018, 15 abr.). Resistencia Kankuama: desde el corazón del mundo. [Video]. YouTube. Recuperado de https://www.youtube.com/ watch? $\mathrm{v}=-$ HilzsypfZw\&t $=57 \mathrm{~s}$

Dussán, A. \& Reichel-Dolmatoff, G. (1977). Estudios antropológicos. Bogotá: Universidad de los Andes.

Dussán, A. \& Reichel-Dolmatoff, G. (2012). La gente de Aritama. Bogotá: Pontificia Universidad Javeriana.

Giddens, A. (2003). La constitución de la sociedad. Bases para la teoría de la estructuración. Buenos Aires: Amorrortu.

Gros, C. (2012). Politicas de la etnicidad. Identidad, Estado y modernidad. Bogotá: ICANH Instituto Colombiano de Antropología e Historia.

Mincultura. (2019, 21 oct.). Pueblo Yanacona. Recuperado de https:/www.mincultura.gov.co/ prensa/noticias/Documents/Poblaciones/PUEBL0\%20YANACONA.pdf

Morales, P. (2011). Los idiomas de la reetnización. Corpus Christi y pagamentos entre los indígenas kankuamo de la sierra nevada de Santa Marta. Bogotá: Universidad Nacional de Colombia. 
Pumarejo, M. A. \& Morales, P. (2003). La recuperación de la memoria histórica de los Kankuamo: un llamado de los antiguos siglos XX-XVIII. Bogotá: Encuentros Universidad Nacional de Colombia.

Ramos, C. A. (2015). Los paradigmas de la Investigación científica. Avances en psicología: revista de la facultad de psicología y humanidades, 23(1), 9-17. https://doi.org/10.33539/ avpsicol.2015.v23n1.167

Ruiz, J. (2009). Análisis sociológico del discurso: métodos y lógicas. Forum Qualitative Social Research FQS, 10(2), 1-32. Obtenido de http:/www.qualitative-research.net/index.php/ fqs/article/view/1298/2777Van Dijk, T. (2009). Discurso y poder. Barcelona: Gedisa.

Van Dijk, T. (2009). Discurso y poder. Barcelona: Gedisa. 